\title{
OBSERVATIONS ON PAIN PRODUCED BY INJECTION OF HY- PERTONIC SALINE INTO MUSCLES AND OTHER SUPPORTIVE TISSUES
}

\author{
By OTTO STEINBROCKER, S. ALLEN ISENBERG, MURRAY SILVER, DAVID \\ NEUSTADT, PAUL KUHN, AND MARILYN SCHITTONE \\ (From the Arthritis Clinics, Bellevue Hospital [4th Medical Division, now of N. Y.U. Post- \\ graduate Medical School] and Lenox Hill Hospital)
}

(Submitted for publication October 17, 1952; accepted June 12, 1953)

To study the clinical effects of experimentally induced pain, as initiated by Kellgren and Lewis, hypertonic saline was injected into selected areas of muscle and about certain tendons and ligaments in a number of individuals (1-3). The resulting subjective sensations and the nature and extent of the objective changes were noted, then tabulated and compared by coworkers not participating in the experiments. This report presents our observations and deductions.

\section{METHOD}

Acute pain was produced by the injection of hypertonic saline solution into various selected anatomic sites. These consisted of the scalenus anticus, supraspinatus, trapezius, deltoid, gluteus medius, and erector spinae muscles; the achilles, sacroiliac interspinous, and insertional biceps tendons, and areas about the knee joint.

A 6 per cent solution of sodium chloride in distilled water was used, as in the investigations of Kellgren (2). The amount of hypertonic saline solution injected at each point varied from 0.2 to $0.5 \mathrm{cc}$.

Eighteen individuals served as subjects for a total of 61 trials. Most of the volunteers submitted to several determinations. The number of trials for each participant is summarized in Table $\mathrm{I}$.

TABLE I

Number of trials per individual

\begin{tabular}{lrrrrrr}
\hline \hline $\begin{array}{l}\text { Number of trials per } \\
\text { individual subject }\end{array}$ & 10 & 5 & 4 & 3 & 2 & 1 \\
\begin{tabular}{l} 
Number of subjects \\
\hline
\end{tabular} & 2 & 1 & 3 & 3 & 6 & 3 \\
\hline
\end{tabular}

This study group consisted of healthy adults who were either physicians, medical students, or nurses. The common medical background reduced to a great extent any emotional factor incident to the use of the needle and syringe.

Subjects were not briefed as to what to expect before the actual testing. Each was required to write immediately a detailed description of such local and referred sensations as occurred. Procaine anesthesia as a preliminary to the infiltration of saline was omitted in order to avoid artificial effects at the point of entry and adjacent areas. Most subjects disregarded the initial needle prick.

Two observers checked each individual for objective sensory and other changes before and after infiltration. Superficial hyperesthesia was determined by pinch, prick, and scratch, and a pressure gage was used to elicit deep tenderness. Comparisons were made each time with symmetrical areas on the opposite side of the body. Alterations in skin temperature were determined by use of the Tycos thermocouple. Careful observations also were made for variations in skin color, sweating, and visible or palpable muscle contraction. The results have been recorded in Table II.

\section{OBSERVATIONS}

The musculosensory responses to the stimuli used by us may be divided into two categories: the primary reaction and certain associated phenomena to be enumerated and discussed later. The primary reaction, the immediate pain at the point of injection, was always present.

The quality of the primary reaction or "local pain" was found to be quite diversified, with no characteristic sensation for any one type of tissue or site of stimulation. The pain at the site of injection was variously described as "sharp," "dull," "severe aching," "throbbing," "stinging," or "burning." This applied to muscle (trapezius), tendon (biceps), and ligament (sacroiliac), as well as to a "complex region" such as the knee joint. It was found, moreover, that even in the same individual the quality of local pain subsequently might change from trial to trial.

The intensity of this local pain was likewise variable, as was its duration, when the same quantity of saline was introduced. Pain or aching at the injection-site lasted from minutes to days, and even recurred in some instances. Cessation usually was gradual, but in some trials at the knee it was strikingly abrupt. 


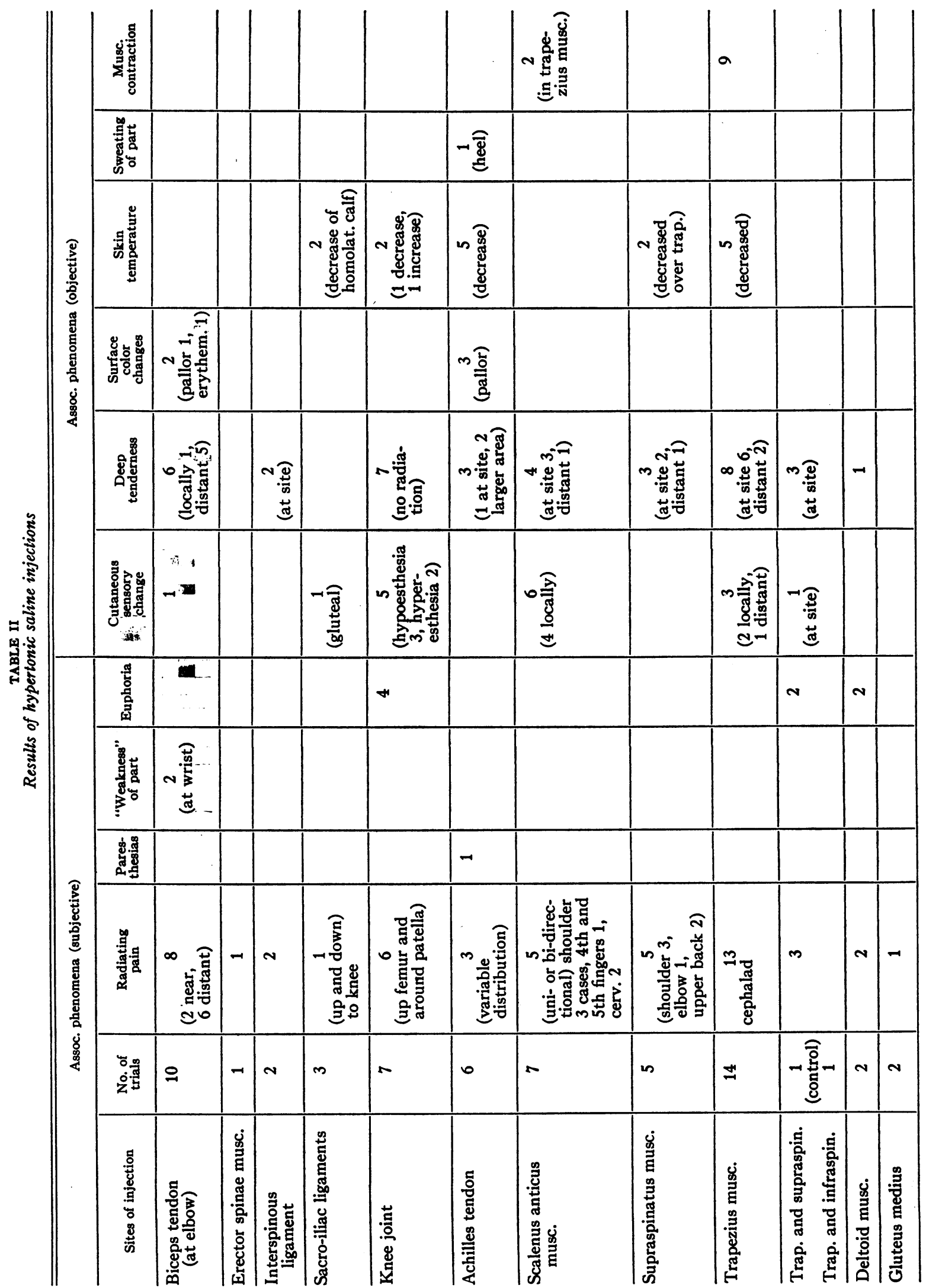


The associated phenomena consisted of a number of subjective and objective reactions accompanying or following the "primary" pain (Figures 1-3). They did not appear in all cases. They were either local (at or over the site of infiltration) or distant, or both, and appeared either as isolated effects or in unpredictable combinations. The reaction patterns were, therefore, rich or meager, and often rather bizarre. Such associated phenomena included both subjective sensations and objective physiologic changes.
Among the subjective reactions were the following : radiating pain of variable quality, intensity and duration even in the same individual given a similar restimulation, paresthesias, a sense of "weakness" of the extremity, and euphoria.

Radiating pain was usually of the same quality as the local pain. Instances wherein these differed were noted at the trapezius and biceps regions. Like the local sensation, the duration of radiation was variable but did not necessarily correspond to it. The direction or pathway of radiating pain was
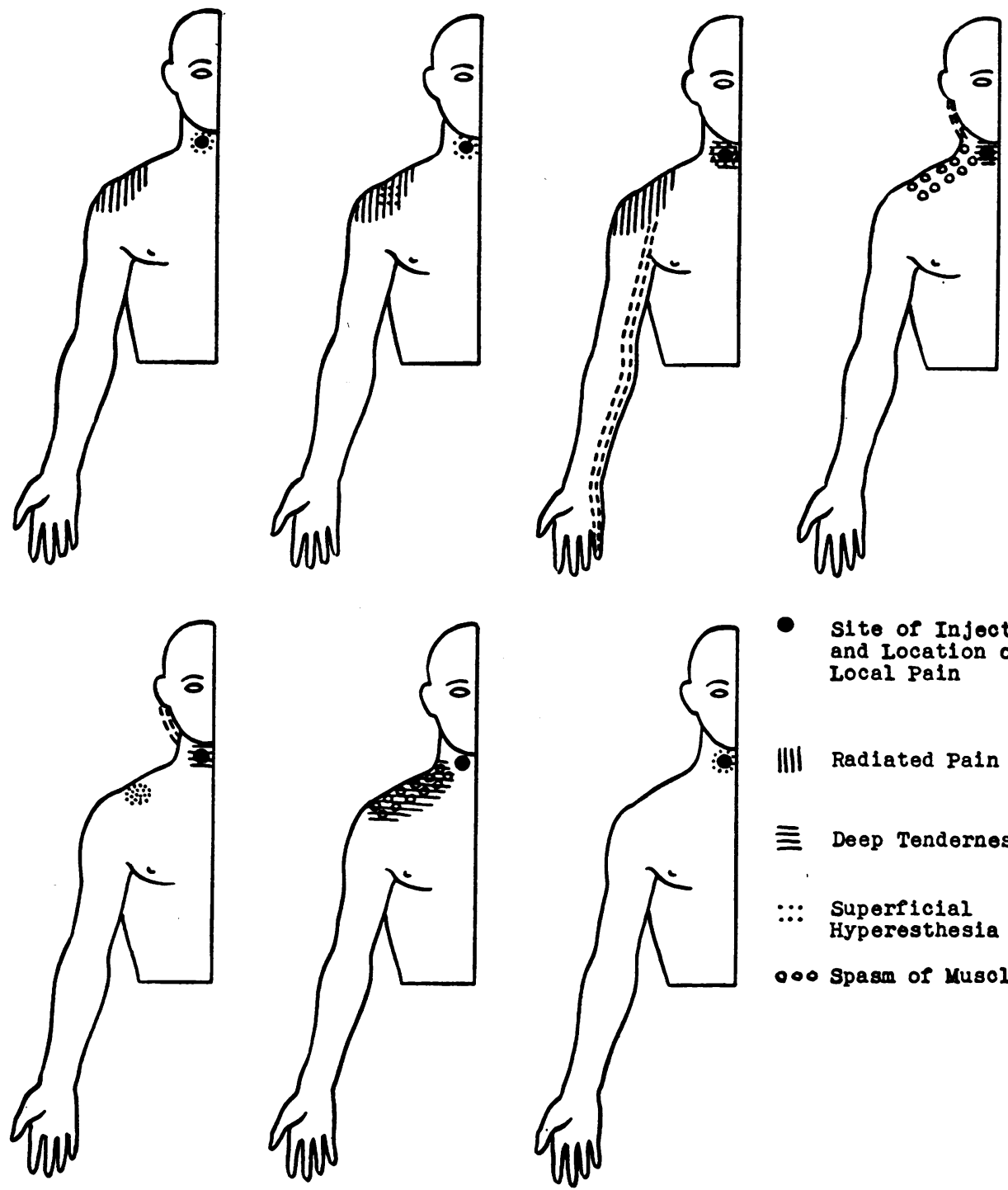

- Site or Injection and Location of Local Pain

IIII Radiated Pain

$\equiv$ Deep Tenderness

$\therefore$ Superficial

Hyperesthesia

-00 Spasm of Musoles

Fig. 1. Scalenus Anticus Muscle

Pattern of pain and associated phenomena following injection of the Scalenus Anticus muscle with 6 per cent Hypertonic Saline in 7 subjects. 

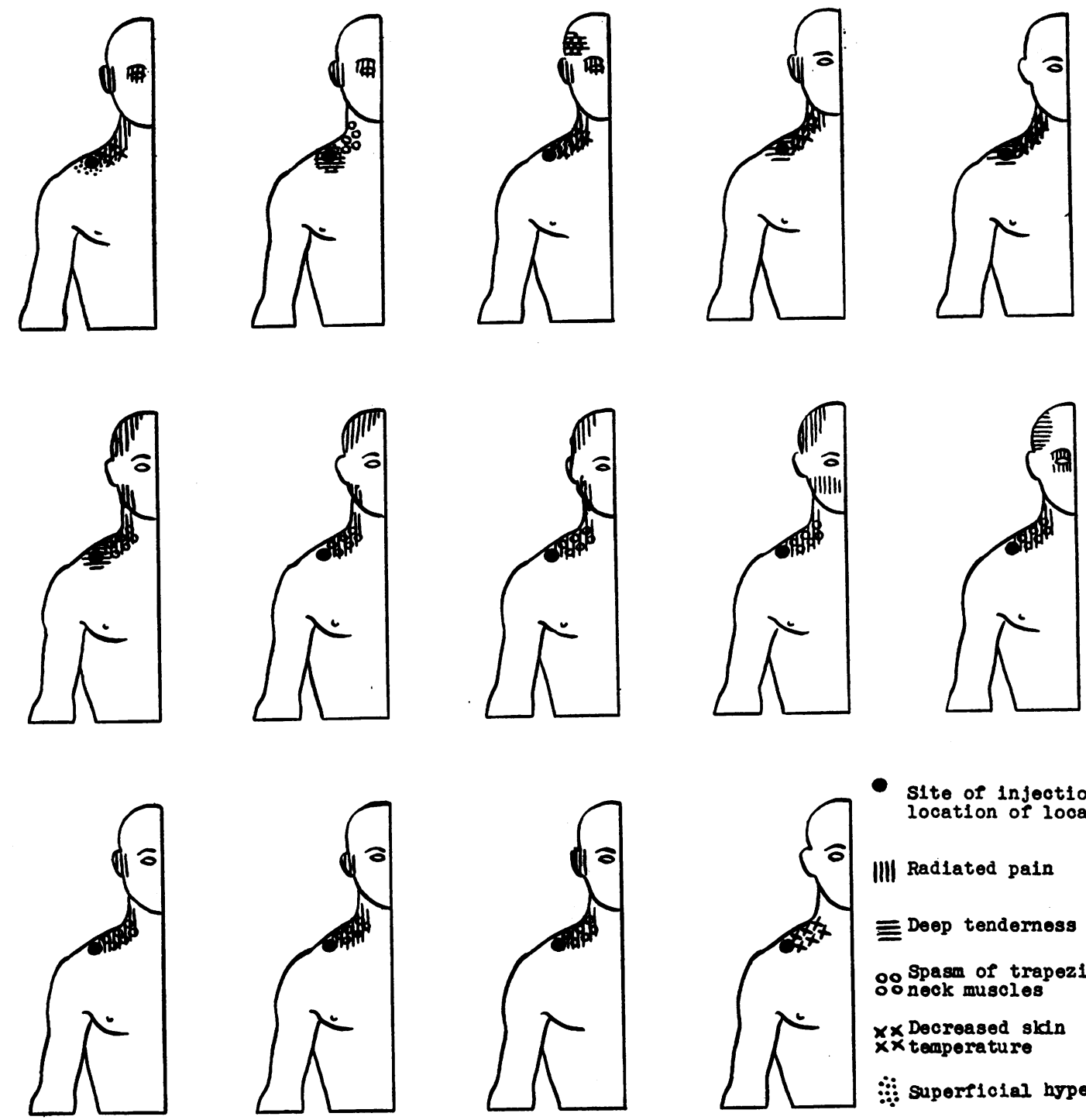

Site of injection and location of local pain

IIII Radiated pain

$\equiv$ Deep tenderness

Oo Spasm of trapezius or o: nock musoles

$x \times$ Decreased sken $\times \times$ temperature

:: superficial hyperesthesia

Fig. 2. Trapezius Muscle

Patterns of pain and associated phenomena following injection of Trapezius muscle with 6 per cent Hypertonic Saline in 14 subjects.

peripheral (descending), central (ascending), or bidirectional. Such pathways often were unrelated to any specific or recognizable neural distribution.

Paresthesias, when associated with radiating pain, frequently affected quite different locations.

In some trials they also included sensations of "warmth" or "burning" which occurred in the absence of objective changes of skin temperature, or even when the area of reference was in reality measurably cooler. This was noted especially after injections of the trapezius and periarticular tissues of the knee.
A subjective "weakness" of the distal part of the upper extremity followed injection of the biceps tendon. This symptom occurred both with and without associated radiating pain.

Euphoria, an unusual "feeling of well-being," was experienced by a few subjects as the induced pain subsided, apparently by contrast.

The local objective changes included superficial hyper- and hypoesthesia, deep tenderness, increased and decreased skin temperature, color changes of flushing and pallor, increased sweating and muscular spasm. 
Changes in superficial sensation and deep tenderness were inconstant in occurrence. The former usually consisted of hyperesthesia, rarely hypoesthesia. The significance of the fact that hypoesthesia was noted only following injection about the knee joint is not clear. The sites of superficial hyperesthesia and deep tenderness involved either the local area (injection site) or distant regions. Both appeared alone or in association with each other. In some instances the area of deep tenderness corresponded to the course of the radiating pain; in other cases it was unrelated.

There was no correlated occurrence of sensory alterations and temperature changes. The latter
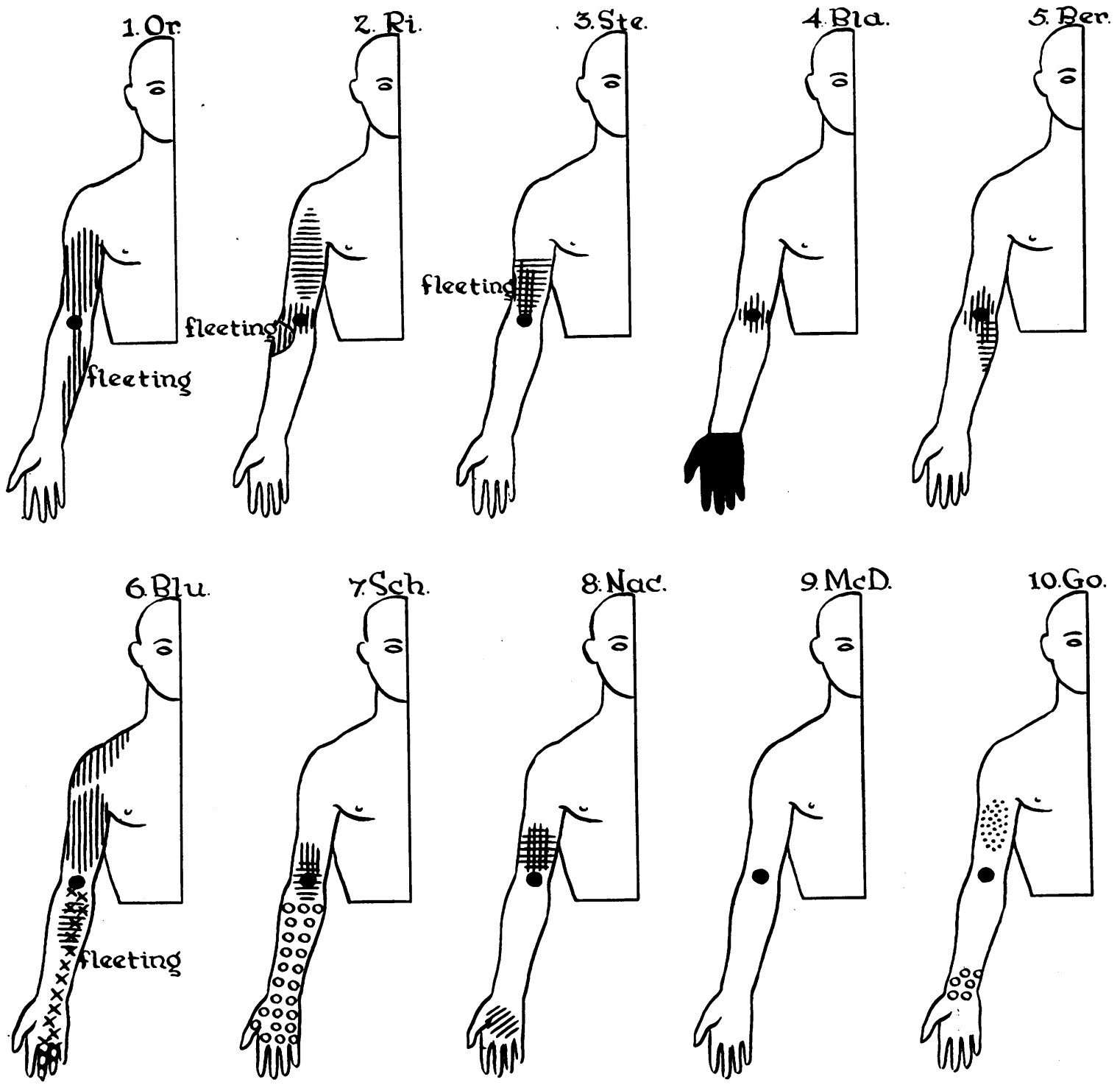

Fig. 3. Biceps Tendon

- Site of injection and location of local pain IIII Radiated pain

三 Deep tenderness

Erythema
: Weakness (subjective)

$\because:$ Superficial hyperesthesia

$\times \times$ Paresthesia

UI, Pallor

Patterns and distribution of associated phenomena following injection of the biceps tendon with 6 per cent Hypertonic Saline solution in 10 subjects. 
also were variable. In the same region (knee) in different subjects the local skin temperature was both increased and decreased. In general, however, the latter reaction predominated.

Color changes, likewise, were diverse. Both pallor and flushing occurred at a distance in different individuals, for example following injection at the biceps tendon. In other instances pallor was the usual reaction. Changes in surface temperature and color did not necessarily appear together.

Sweating, like color change, was observed at a distance from the injection site.

It is of interest that injections were followed by visible or palpable muscular contraction only in the neck and shoulder regions.

\section{DISCUSSION}

In our hands the reactions of supportive tissues to the infiltration of hypertonic saline solution obviously were varied and unpredictable in quality and quantity for each site. In fact in some instances the effects were different even when the procedure was repeated later at a chosen location in the same individual. Our observations show that in some subjects the injection of hypertonic saline into certain supportive structures produces local pain without any demonstrable coincidental effects. In others, however, any of a variety of associated reactions may appear. The pain and associated phenomena suggested a definite segmental or a specific neural pattern in only a few of our trials, in contradistinction to the observations of Kellgren and Lewis. In most of our trials, in fact, the reactions were too complex or too irregular for the identification of typical pathways. Perhaps a larger number of injections of spinal ligaments would provide more data conforming to the patterned segmental distributions deduced by Kellgren and Lewis.

The remarkable diversity of responses elicited by the same stimulus at corresponding sites naturally has raised questions in our minds as to the sources of these variations. To interpret this medley of reaction-patterns it is possible that, in addition to a small number of trials suggestive of segmental distribution, some represent an axone reflex, others the direct stimulation of a peripheral nerve filament or possibly of a sympathetic element. Finally, in a small number there appears to be stimulation of higher or lower segments without evidence from the direct level of irritation. The nature, severity and distribution of response to the stimulus, therefore, would seem to depend on its greater or lesser proximity to any or several of the multiple constituents of the infiltrated tissuenerve element(s) (spinal or sympathetic-trunk, fibril, or end-organ); blood vessel(s) (trunk or branch, sheath or other layer); fascia, muscular bundles, ligament, subcutis, or periosteum. Whether or not these considerations account for the variability of our observations, the experimental results and the difficulties of interpreting them do not permit some of the generalizations and applications previously derived from similar investigations $(2,3)$.

A noteworthy observation during these experiments was the absence of any "trigger" mechanisms in the reactive phenomena produced. Palpation at the site of injection or other tender areas, immediately after the infiltration or while discomfort lasted, usually produced local soreness. In no instance, however, did it quite provoke the related features with the pain initially associated with the infiltration. It seems likely that the nature of the stimulus and the effects it aroused were inadequate or too brief in their action to create the "trigger points" and their clinical patterns ascribed to some muscular, myofascial, and musculoskeletal disorders (5-11).

The injection of hypertonic saline into the various supportive tissues in our series has not produced a distinctive reaction-pattern for each anatomic unit which might thereby be recognized clinically, as Kellgren's studies imply. These observations do not provide a basis for the current trend toward the delineation of symptom-complexes or "syndromes" characteristic of lesions of various supportive structures, particularly muscles. Stimulation with hypertonic saline usually evokes rather temporary effects, sometimes quite explosive in-intensity and spread. These suggest to the observer, as the most closely analogous clinical situations, the acute and transient symptoms of traumata, or of a moderately potent insect bite. It is quite doubtful whether such acute reactions and their effects may be compared correctly with chronic disorders in which the acute features have subsided and the persistent disturbance and structural changes create physiologic mechanisms and 
clinical patterns largely dependent on the factors of time and prolonged stimulation.

Our observations, therefore, indicate that injections of hypertonic saline may be regarded as an adequate stimulus only for the production of acute and transient pain and related phenomena. Furthermore, these responses in our series have neither provided a factual basis for deducing a specific segmental or neural pathway for each anatomic entity stimulated, nor have they constituted distinctive, consistent reaction patterns by which involvement of each structure might be identified in clinical diagnosis.

\section{SUMMARY AND CONCLUSIONS}

1. The clinical effects of pain experimentally induced by the injection of hypertonic ( 6 per cent) solution of sodium chloride, as initiated by Kellgren and Lewis, were studied in 18 volunteers subjected to 61 trials.

2. The material was injected into muscles-the scalenus anticus, supraspinatus, trapezius, deltoid, gluteus medius, and the erector spinae; into or about tendons-the achilles and insertional biceps; at ligaments-the sacroiliac and interspinous and about the knee joint.

3 . Injection of hypertonic saline into muscle, about a tendon, or at a ligament caused pain and aching at the point of infiltration, usually associated with one or more of the following phenomena; radiating soreness or shooting pain at a distance, paresthesias, hyperesthesia of overlying skin, and/ or at a farther area, greatest tenderness to palpation at the point of injection, lesser soreness to palpation at a distant region, muscle "spasm," stiffness, and increased or decreased cutaneous temperature.

4. These complex but variable reactions were immediately or quickly provoked. Aching at the point of injection lasted for minutes to days. The other features disappeared rapidly when present.

5. Our data may be said to confirm the findings of Kellgren and Lewis only to the extent that pain always, and a local point of greatest soreness often, were induced at the site of injection. These were accompanied only at times by an area of lesser tenderness of skin or muscle about it or at an adjacent or distant location.

6. In some cases the demarcations of pain and tenderness suggested a segmental distribution. In most instances these features were irregular and without the definite segmental or recognizably uniform characteristics described by the original observers.

7. Consistent similar effects from stimulation of each of the various selected sites were too infrequent to deduce predictable clinical reaction patterns typical of individual anatomic structures.

8. Our observations on the acute effects of injecting hypertonic saline failed to provide an experimental basis for such clinical concepts in musculoskeletal pain as "trigger point" mechanisms or specific, diagnostic reaction patterns to identify involvement of individual muscular and other supportive structures.

\section{REFERENCES}

1. Lewis, T., Suggestions relating to the study of somatic pain. Brit. M. J., 1938, 1, 321.

2. Kellgren, J. H., A preliminary account of referred pains arising from muscle. Brit. M. J., 1938, 1, 325 ; On the distribution of pain arising from deep somatic structures with charts of deep segmental pain areas. Clin. Sc., 1939, 4, 35; Somatic simulating visceral pain. Clin. Sc., 1940, 4, 303.

3. Lewis, T., Pain, New York, Macmillan Co., 1942.

4. Gage, M., Scalenus anticus syndrome. A diagnostic and confirmatory test. Surgery, 1939, 5, 599.

5. Steindler, A., and Luck, J. V., Differential diagnosis of pain low in the back. J.A.M.A., 1938, 110, 106.

6. Livingston, W. K., Pain Mechanisms, A Physiologic Interpretation of Causalgia and Its Related States, New York, Macmillan Co., 1943.

7. Judovich, B., and Bates, W., Segmental Neuralgia in Painful Syndromes, Philadelphia, Davis, 1944.

8. Gorrell, R. L., Musculofascial pain. Treatment by local injection of analgesic drugs. J.A.M.A., 1950, $142,557$.

9. Good, M. G., Diagnosis and treatment of low backache. Med. Press, 1948, 220, 498.

10. Travell, J., Basis for the multiple uses of local block of somatic trigger areas. (Procaine infiltration and ethyl chloride spray.) Mississippi Valley M. J., 1949, 71, 13; Travell, J., and Rinzler, S. H., The myofascial genesis of pain, a scientific exhibit. Postgrad. Med., 1952, 11, 425.

11. Steinbrocker, O., Arthritis in Modern Practice; The Diagnosis and Management of Rheumatic and Allied Conditions. Philadelphia, Saunders, 1941. 